\title{
Constitución económica, autonomía de la voluntad y figuras financieras disruptivas. El caso concreto de las Initial Coin Offering (ICO)
}

Economic constitution, autonomy of the will and disruptive financial figures.

The concrete case of the «Initial Coin Offering» (ICO)

El núcleo fundamental del trabajo lo constituye la figura de las Initial Coin Offering como nueva forma de financiación tecnológica y global. Se dedicarán unas líneas iniciales al contenido económico y financiero de nuestra Constitución. Nos planteamos si el control público de la negociación en los mercados financieros supone un fenómeno reductor del principio de autonomía de la voluntad. Con objeto de dilucidar hacia dónde nos dirigimos señalaremos las actuaciones recientes de las autoridades supervisoras -tanto en el ámbito comunitario como en el nacional- en relación con el fenómeno disruptivo de las ICO. Es nuestra intención concluir planteando una serie de retos de futuro que puedan ser de utilidad para los operadores del tráfico.

PALABRAS CLAVE: derecho civil, derecho constitucional, derecho mercantil, mercado financiero.

In this paper the current regulatory situation and attitudes amongst competent authorities are analysed in relation with the Initial Coin Offerings (ICO) phenomenon. The phenomenon is understood as a new form of financing with global coverage. It is considerated in relation to the principle of party autonomy. Financial and economic content of our Constitution is noted. Finally, legal challenges will be highlighted.

KEYWORDS: civil law, commercial law, constitutional law, financial markets.

Este trabajo tiene su origen en una investigación iniciada en el seno del grupo de investigación interuniversitario BAES Blockchain ${ }^{1}$, parte del cual está pendiente de publicación en la Revista de Derecho del Mercado de Valores.

1. Presentado el 19 de octubre de 2018 en el Colegio Notarial de la Comunidad Valenciana, en la delegación de Alicante, en la Jornada Técnica de Debate: «¿Necesitamos un marco legal para Blockchain?». 
Dada la repercusión económica y jurídica del fenómeno se detectó la necesidad de avanzar y elevar la cuestión objeto de investigación a ámbitos más generales de la contratación, con el fin de ponerla en relación con el principio imperante de autonomía de la voluntad de las partes que rige en nuestro Derecho.

Por otro lado, no podríamos dejar de señalar la coincidencia de este estudio con el 40 aniversario de nuestra Constitución. Esta razón nos lleva a hacer referencia, para que sirva como homenaje, al contenido económico de la misma.

\section{Introducción}

Asistimos a una revolución tecnológica que avanza a un ritmo vertiginoso en casi todos los sectores. Para entender lo que supone este cambio brusco y disruptivo es necesario analizar la digitalización en el mundo empresarial. Siguiendo a Ramos Suárez (2018), cuando un sector empresarial o industrial se digitaliza tiende a «desmaterializarse, democratizarse y desmonetizarse». La desmaterialización se produce con la digitalización del producto, que queda a disposición del usuario únicamente en el mundo digital; la democratización permite que cualquier persona con conexión a Internet acceda a lo mismo, y, por último, la desmonetización supone una reducción considerable de costes como consecuencia de la digitalización.

En este sentido, Pastor Sempere (2017) afirma que puede llegar a considerarse que estamos asistiendo a una revolución impulsada por medios digitales y tecnológicos, que se está difundiendo a una velocidad sin precedentes gracias a los millones de usuarios interconectados en todo el mundo a través de Internet y de las nuevas tecnologías.

Centrémonos en el sector financiero. Esta irrupción tecnológica ha impulsado nuevas formas de financiación alternativas a la bancaria. Muñoz Pérez (2018) considera que estas se dirigen a la financiación de proyectos empresariales de mediana y pequeña dimensión, particularmente en el sector de las nuevas tecnologías. A través de las «rondas de financiación", participan inversores por medio de las operaciones ICO, las venture capital y crowdfunding.

Son muchos los aspectos susceptibles de estudio para establecer su integración en nuestro sistema. Por las características del trabajo que desarrollamos, nos limitaremos a considerar uno de ellos con clara connotación jurídica: el encaje en nuestro sistema de las plataformas, concretamente ICO, que actualmente están provocando un cambio de paradigma y superando los tradicionales parámetros de la empresa capitalista. Este nuevo paradigma se basaría en los principales componentes de la economía digital: intercambio de criptodivisas, plataforma de comercialización y plataforma ICO (Pastor Sempere, 2017).

La Carta Magna y la completa integración en Europa constituyen los fundamentos sobre los que se asienta, en general, ese proceso de adecuación de nuestras instituciones económicas y financieras a estas nuevas figuras. Desde la promulgación de la Constitución española, el sistema financiero ha experimentado profundas transformaciones, gran parte de ellas coadyuvadas por el fenómeno disruptivo de las nuevas tecnologías. La búsqueda de la constante homologación con los países más avanzados de nuestro entorno ha sido clave en este desarrollo. 


\section{Apunte sobre contenido económico y financiero de nuestra Constitución en materia de mercado}

En un trabajo publicado con motivo del 25 aniversario de la promulgación de nuestra Constitución, los profesores Ontiveros Baeza y Valero López (2003) ya advertían que la propia evidencia disponible permitía observar que difícilmente puede atribuirse a la Constitución española una influencia clara y directa sobre las transformaciones del sistema financiero.

Aun siendo portadora de un contenido político más que económico, nuestra Constitución contiene algunos artículos que, de alguna forma, pueden caracterizarla como "constitución económica». En nuestra opinión, dentro de la Constitución de 1978, los artículos concretos de mayor relevancia en relación con el modelo de constitución económica son los artículos 38, 128 y 131, al constituir la clave para la determinación del tipo de marco de relaciones económicas fijadas por nuestra norma fundamental.

Sin tratarse de la figura jurídica esencial para el proceso de transformación, la Constitución ha facilitado la incorporación de nuestro país en un marco europeo más amplio, y por consiguiente, ha hecho posible una mayor apertura económica y financiera hacia el exterior; en este sentido, puede atribuirse a la misma el influjo sobre las sucesivas transformaciones que han acontecido en lo que a formas de financiación se refiere.

No puede olvidarse que las bases de crédito, banca y seguros se encuentran condicionadas, estrecha y directamente, por disposiciones del mercado único europeo de servicios financieros, que, si bien no eliminan toda la competencia nacional en este ámbito, sí la someten a un proceso de armonización de sus principales elementos básicos a nivel europeo (Ontiveros Baeza y Valero López, 2003).

\section{Breve reflexión: ¿constituye el control público de la negociación en los mercados financieros un fenómeno limitador de la autonomía de la voluntad?}

Visto que nuestra Carta Magna no ha supuesto cortapisa en el avance de los mercados financieros, sino que se ha limitado a garantizar la apertura de estos al espacio europeo, nuestro siguiente objetivo es relacionar el control público de dichos mercados financieros con el principio de autonomía de la voluntad de las partes que rige en Derecho de contratos.

Centraremos la reflexión en el sector relativo a mercado de valores, por restricción de espacio y por constituir la vertiente más afín al fenómeno de las ICO en el que profundizaremos más adelante.

Es a todas luces evidente la conmixtión entre normas administrativas y mercantiles en sedes de mercado de valores. Base del funcionamiento de este mercado son también las normas comunes del Derecho de obligaciones y contratos.

Desde sus orígenes, han sido razones de interés general las que han propiciado la conveniencia de una ordenación pública de este sector del Ordenamiento. Ibáñez Jiménez (2001) considera que en el actual contexto de economías mixtas, relativamente desreguladas 
y con creciente recurso a la autorregulación por intermediarios de valores y otras empresas de servicios de inversión, parece inevitable que los mecanismos autodispositivos, amparados por el propio Ordenamiento, se vean encauzados, evaluados y supervisados.

En este sentido, el Real Decreto Legislativo 4/2015, de 23 de octubre, por el que se aprueba el texto refundido de la Ley del Mercado de Valores (en adelante, TRLMV), en sus artículos 17 y 21 atribuye a la Comisión Nacional del Mercado de Valores (en adelante, CNMV) competencias en materia de supervisión e inspección de los mercados de valores y de la actividad de cuantas personas físicas y jurídicas se relacionan en el tráfico de los mismos, así como el ejercicio sobre ellas de la potestad sancionadora.

Para el adecuado ejercicio de las competencias que hemos referido, podrá dictar las disposiciones que exija el desarrollo y ejecución de las normas contenidas en los reales decretos aprobados por el gobierno o en las órdenes del ministro de economía y competitividad, siempre que estas disposiciones le habiliten de modo expreso para ello.

Dichas competencias, unidas a la potestad regulatoria que se le atribuye para su efectivo ejercicio, podrían llevarnos a pensar en una hipotética limitación de la libre voluntad de los contratantes en aras de garantizar la libertad de mercado y el equilibrio en la contratación, evitando abusos de mercado que afecten a los inversores. Ahora bien, esta idea debe ponerse en relación con el debido análisis de la actividad de la CNMV "caso por caso», ya que, en general, estas actuaciones administrativas supondrán una prevención ex ante y una curación ex post de determinados fallos inherentes al mercado.

En su reflexión sobre esta materia, el profesor Ibáñez Jiménez (2001) llega a la conclusión, que compartimos, de que los mecanismos correctores de la libertad de mercado resultan imprescindibles para asegurar el funcionamiento eficiente de los propios mercados y tutelar la autonomía de la voluntad de quienes promueven la relación de negocios jurídicos en los mismos.

\section{ICO: nueva forma de financiación tecnológica y global}

Una vez planteadas las cuestiones de índole constitucional y otras de conmixtión de Derecho público y Derecho privado de contratos, nos centramos en la figura sobre la que gira este estudio: las «ofertas iniciales de monedas», en su traducción literal al castellano, como fenómeno disruptivo de financiación alternativa a las tradicionales fórmulas de financiación bancaria.

En sentido amplio y tratando de simplificar la definición al máximo, puede entenderse que una ICO es esencialmente una forma de financiación participativa que, mediante el uso de un registro virtual distribuido o de tecnología Blockchain, permite a promotores de proyectos financiarse por medio de la emisión de «tokens» digitales. Los inversores interesados en estos proyectos realizan la aportación establecida para esa ICO, que podrían ser monedas digitales convertibles, como Bitcoins o Ethers, a cambio de los «tokens» que, a su vez, representarán las prestaciones futuras que ofrece el promotor (González Echenique, 2017).

La conceptualización que de ICO se hace desde la perspectiva de Ethereum es muy similar. Define la ICO como evento en el que un proyecto basado en Blockchain vende una 
serie de «tokens» a los primeros usuarios a cambio de criptomonedas. Esto significa que el proyecto Blockchain ofrece a los inversores algunas unidades de una nueva criptomoneda (su «token») a cambio de otras criptomonedas más conocidas como pueden ser Bitcoin o Ethereum. Nos encontramos, por tanto, ante un método de captación de fondos que se basa en el intercambio de futuras monedas digitales por monedas digitales ya existentes que cuentan con liquidez.

Referimos, por último, el concepto al que se acogen las entidades supervisoras. Entienden que las ICO se presentan como una nueva vía de captar fondos del público, utilizando criptomonedas o «tokens». Una ICO también puede ser denominada como una «oferta inicial de tokens» o «venta de tokens». En una ICO, una compañía o una persona emite monedas o «tokens» y las pone a la venta para intercambiar por divisas tradicionales, como el euro, o más comúnmente por criptomonedas, como el Bitcoin o el Ether. Las funcionalidades y usos de las monedas o «tokens» varían según las ICO. Algunas monedas o «tokens» sirven para acceder o comprar un servicio o producto que el oferente desarrolla utilizando la tecnología de registro descentralizado. Otras otorgan derechos de voto o participación en los derechos futuros de la empresa emisora. Y otras, en cambio, no tienen un valor tangible. Algunas de estas monedas o «tokens» son negociadas y/o pueden ser intercambiadas por monedas tradicionales o virtuales en casas de cambio especializadas. Las campañas de ICO se realizan online, utilizan Internet y las redes sociales. Las monedas o «tokens» son normalmente creadas y diseminadas mediante tecnologías de registro descentralizado o Blockchain. Las ICO son utilizadas con el fin de recaudar fondos para una variedad de proyectos, incluyendo, aunque sin limitarse, al aprovechamiento de negocios basados en DLT. Virtualmente cualquiera que tenga acceso a Internet puede participar en una ICO.

Antes de continuar, conviene clarificar qué se entiende por «token». Seguimos el criterio de aportar definiciones sencillas y adaptadas al contexto ICO en el que trabajamos. El «token» representa la unidad de valor que se oferta al mercado destinatario de la «emisión» a cambio de la inversión realizada para que el oferente pueda acometer un determinado proyecto empresarial. En opinión de García Rodríguez (2017) los derechos inherentes a esa unidad de valor pueden ser de distinta naturaleza. Simplificando al máximo las clases de «tokens», puede considerarse la existencias de dos tipos: «tokens» emitidos que representan capital o deuda de la puesta en marcha de la empresa, denominados security tokens; y los utility tokens, que representan un derecho de uso de un producto o servicio en el ecosistema del proyecto financiado.

Adelantamos que las ICO no se encuentran expresamente reguladas en nuestro país; pese a ello, la CNMV, como el resto de supervisores europeos e internacionales, es consciente de la dificultad que puede entrañar el encaje de los instrumentos que se emiten en estas operaciones en las normas vigentes, así como de la posible falta de adecuación del marco regulatorio a algunos nuevos modelos de negocio y colaboración digital. Sin embargo, ya comienzan a darse los primeros indicios de que sí serán instrumentos de financiación específicamente regulados en los próximos años ${ }^{2}$.

2. Conviene tener en cuenta también que numerosas autoridades del mercado de valores de diferentes países se han pronunciado expresamente sobre este sector y han considerado que estas emisiones de criptomonedas pueden calificarse como emisiones de valores y, por tanto, se les aplicaría la normativa del mercado de valores del país donde se realiza la emisión. 
Uno de los atractivos fundamentales, y que podría considerarse la clave de su éxito, es la reducción de costes transaccionales al inicio del proyecto que se pretende financiar. Se encuentra materializado en términos de ahorro de tiempo, permite el acceso a financiación en fase prácticamente pre-proyecto y sin las clásicas exigencias de defensa y documentación del mismo ante las tradicionales entidades bancarias.

Profundicemos en la propia emisión ICO. Tomamos para nuestro ejemplo las tecnologías más frecuentes: Ethereum y Bitcoin. A través de ellas, se emite un «token». El inversor recibirá este «token» y el emisor, a cambio, recibirá una moneda líquida y de valor en el mercado: Bitcoins o Ethers.

Para la toma de decisión, el inversor, debe estudiar el plan de negocio que se implementará, y que estará reflejado en un folleto o white paper. Lo habitual es que los emisores se presenten y justifiquen la viabilidad de su plan de negocio así como la necesidad de la financiación que están solicitando. Se establecerá un plazo durante el cual se puede adquirir el «token». Finalizado dicho plazo, la adquisición del «token» o criptomoneda se materializa mediante la recepción de un documento en formato PDF en el que se informa al inversor de que, una vez completado el proceso de implementación de la idea de negocio, los inversores tendrán acceso a los servicios ofertados o recibirán la criptomoneda para cuya emisión se solicitó financiación.

De ahí en adelante, entra en juego el compromiso del emisor de implementar la idea que constaba en el proyecto. A la espera de los resultados del mismo, el valor del «token» se encontrará supeditado a la especulación; puede crecer exponencialmente o devenir ilíquido.

\section{Actuaciones de autoridades supervisoras}

Proseguimos nuestra exposición refiriendo actuaciones de las autoridades supervisoras a escala europea y nacional, Autoridad Europea de Valores y Mercados (en adelante, AEVM) y CNMV, respectivamente. Procedemos conforme a cronología de actuaciones. Lo hacemos en principio con una mera intención enunciativa, para más adelante plantear retos y esbozar ideas de futuro.

\subsection{Noviembre 2017. AEVM se dirige a inversores y promotores en relación con las ICO}

Con fecha 13 de noviembre de 2017, AEVM publica dos comunicados sobre las colocaciones en el mercado de las criptomonedas o «tokens" y las ICO, uno relativo a los riesgos para el inversor y otro a las normas específicas aplicables a las empresas que participan en ellas.

Con fecha 14 de noviembre de 2017, CNMV se hace eco del contenido a través de nota de prensa. Manifiesta que ha participado en el análisis realizado por ESMA y pone a disposición de las partes interesadas un canal online para resolver las dudas del inversor y otro para las entidades o promotores.

Señalemos a continuación las notas fundamentales del contenido de estos informes. 


\section{Comunicado dirigido a inversores}

AEVM manifiesta su preocupación debido a que los inversores no son conocedores del elevado riesgo que estarían asumiendo al participar en ellas, dado que las ICO son inversiones muy especulativas y que, dependiendo de cómo se estructuren, podrían no enmarcarse en la regulación de la UE. Refiere el riesgo de fraude o de blanqueo de capitales, debido a su anonimato y a su capacidad de recaudar altas cantidades de dinero en un breve periodo de tiempo.

Enumera los riesgos que deben ser conocidos por los inversores: están fuera del espacio regulado, son vulnerables al fraude o actividades ilícitas, existe elevado riesgo de perder todo el capital invertido, disponen de pocas opciones de reembolsar la inversión y poseen una extrema volatilidad del precio de las monedas o «tokens", suponen un riesgo de información inadecuada y pueden darse fallos en la tecnología al tratarse de tecnología en periodo de pruebas.

\section{Comunicado dirigido a promotores}

El objetivo de este informe fue enfatizar el hecho de que los promotores deben prestar especial atención en comprobar si realizan o no actividades reguladas. Explica que es deber de las entidades considerar el marco regulatorio, solicitando los permisos necesarios y adaptándose a los requerimientos aplicables. Para ello recoge un resumen general de la legislación clave de la UE que sería aplicable.

- Directiva sobre folletos: tiene como objetivo asegurar que las compañías entreguen la información adecuada a los inversores cuando piden fondos al público en la UE. En cuanto al ámbito objetivo, requiere la publicación de un folleto antes de la oferta de transmisión de valores al público o de la admisión de la negociación de dichos valores en un mercado regulado determinado o que opera dentro de un Estado miembro, salvo para ciertas exclusiones o exenciones. Especifica que el folleto debe contener la información necesaria, la cual es el material con que cuenta un inversor para hacer una evaluación informada de los hechos, y esa información debe ser presentada de una manera comprensible y fácil de analizar. La directiva sobre folletos no especifica quién debe redactarlo, pero requiere que la parte responsable de la información (siendo el emisor el oferente, la parte que solicita la admisión de negociación o el garante) se especifique en el folleto. Dependiendo de cómo esté estructurada la ICO, las monedas o «tokens» pueden, potencialmente, entrar dentro de la definición de valor transferible, y podría, por lo tanto, necesitar que la publicación del folleto esté sujeta a la aprobación de una autoridad competente.

- En el momento de la publicación del informe por ESMA, la referencia procedente sería a MiFID I (actualmente vigente MiFID II). Directiva 
sobre Instrumentos Financieros. Esta directiva establecía los requisitos que debían cumplir las entidades que proveen actividades y/o servicios de inversión en relación con instrumentos financieros. En el caso de las ICO, donde la moneda o «token» es creada, distribuida o negociada, es probable que involucre alguna actividad/servicio MiFID, como la colocación, negociación o asesoramiento en instrumentos financieros. Los requisitos de organización, las reglas de conducta y los requisitos de transparencia establecidos en MiFID les serían de aplicación.

- Directiva sobre Gestores de Fondos de Inversión Alternativos: esta directiva establece las normas para la autorización, sus operaciones habituales y la transparencia con que deben operar los gestores de fondos de inversión alternativos que gestionan este producto en la UE. Dependiendo de cómo esté estructurada, el esquema de una ICO puede calificarse como una gestora de fondos de inversión alternativos, en la medida en que se utilice para recaudar fondos de varios inversores, con el fin de invertir de acuerdo a una política de inversión definida. Las entidades que participen en este tipo de ICO necesitan, por lo tanto, cumplir con la reglamentación de la mencionada directiva. En particular, proporciona reglas sobre capital, operativas y organizativas y requisitos de transparencia.

- Cuarta Directiva Antiblanqueo de Capitales: tiene como objetivo prohibir el blanqueo de capitales y la financiación al terrorismo. La directiva exige a estas entidades llevar a cabo un análisis exhaustivo sobre sus clientes, además de un mantenimiento apropiado de registros y de otros procedimientos internos. Las entidades tienen la obligación de reportar cualquier actividad sospechosa y cooperar con cualquier investigación realizada por las autoridades públicas competentes.

\subsection{Enero 2018. La CNMV se hace eco del comunicado de la SEC}

La CNMV se hace eco, a través de su enlace y traducción, del comunicado emitido por la Securities and Exchange Commission (Comisión del Mercado de Valores de los Estados Unidos) sobre criptomonedas e ICO. Nuestra autoridad supervisora consideró que, aunque los entornos normativos y de mercado español y estadounidense presenten diferencias significativas, se estima que las consideraciones, conclusiones y recomendaciones contenidas en el comunicado pueden ser una guía útil para inversores y profesionales del sector.

Parte de la afirmación de que las plataformas mundiales de redes sociales y mercados financieros están en ebullición debido a la proliferación de criptomonedas y de ICO. El comunicado proporciona la propia visión general de quien lo redacta. Recoge consideraciones para inversores minoristas y profesionales del mercado.

En relación con las consideraciones para minoristas, señalamos:

«Han surgido preocupaciones con respecto a los mercados de criptomonedas y las ICO, incluyendo que, tal y como están operando en la actualidad, hay considerablemente menos 
protección para los inversores que en nuestros mercados de valores tradicionales, con los consiguientes mayores riesgos de fraude y manipulación».

«Como con cualquier otra inversión potencial, si un promotor garantiza ganancias, si una oportunidad suena demasiado buena para ser verdad, o si es presionado para actuar rápidamente, por favor, extreme la precaución y sea consciente del riesgo de perder su inversión».

«Por favor, tenga en cuenta también que estos mercados cruzan las fronteras nacionales y que puede haber negociación en sistemas y plataformas situadas fuera de los Estados Unidos. Sus fondos invertidos pueden moverse rápidamente hacia el extranjero sin su conocimiento. Consecuentemente, los riesgos pueden ir aumentando, incluyendo el riesgo de que los reguladores de mercado, como la SEC, no puedan perseguir de manera efectiva a actores fraudulentos o recuperar el capital invertido».

Y en relación con los profesionales del mercado:

«Advierto también a los participantes del mercado en contra de promocionar o publicitar la oferta y venta de criptomonedas sin determinar primero si las leyes de valores se aplican a esas actividades. Vender valores generalmente requiere una licencia, y la experiencia demuestra que una excesiva promoción en mercados poco activos y volátiles puede ser un indicador de estrategias de scalping, hinchar y pinchar (pump and dump) y otras manipulaciones o fraudes. Al mismo tiempo, también advierto a quienes operan en sistemas y plataformas que efectúan o facilitan transacciones en esos productos que pueden estar gestionando exchanges (mercados) o brokers-dealers no registrados, infringiendo la Ley del Mercado de Valores de 1934 ».

«Antes de lanzar una criptomoneda o un producto con su valor vinculado a una o más criptomonedas, sus promotores deben (1) ser capaces de demostrar que la moneda o producto no es un valor o (2) cumplir con el registro aplicable y otros requisitos bajo nuestras leyes de valores. Segundo, los brokers-dealers y otros participantes del mercado que permiten pagos en criptomonedas, consintiendo a los clientes comprar criptomonedas apalancadas, o que utilicen criptomonedas para facilitar transacciones de valores, deben tener una especial precaución, incluyendo el asegurarse de que las actividades de sus criptomonedas no están socavando las obligaciones de blanqueo de capitales y las de identificación del cliente. Como he dicho anteriormente, estos participantes del mercado deben tratar los pagos y otras transacciones hechas en criptomonedas como si fuera efectivo entregado de una parte a otra».

Además, incluye una discusión adicional sobre criptomonedas, ICO y regulación de valores. Para concluir, manifiesta el compromiso de la SEC de promover la formación de capital. Anima a los inversores minoristas a abrirse a esas oportunidades, pero pidiendo información, exigiendo respuestas claras y aplicando el sentido común cuando lo hagan, e insta a los profesionales a considerar cuidadosamente leyes, regulaciones y guías.

\subsection{Febrero 2018. Consideraciones de la CNMV sobre «criptomonedas» e ICO dirigidas a los profesionales del sector financiero}

La CNMV manifiesta en su comunicado que es consciente de la dificultad que puede entrañar el encaje de los instrumentos que se emiten en estas operaciones en las normas vigentes, 
así como de la posible falta de adecuación del marco regulatorio a algunos nuevos modelos de negocio y colaboración digital. Advierte de la necesidad de un esfuerzo de coordinación internacional y reconoce los beneficios que pueden aportar el desarrollo tecnológico y la innovación en los servicios financieros, comprometiéndose a tener en cuenta al ejercer sus funciones de supervisión en este ámbito el principio de proporcionalidad.

Dice expresamente que buena parte de las operaciones articuladas como ICO deberían ser tratadas como emisiones u ofertas públicas de valores negociables. Ello con base, entre otras razones, en el amplio concepto de valor negociable contenido en el artículo 2.1 del TRLMV. Señala que ha de tenerse en cuenta en particular en aquellas ICO en las que el «token» atribuya derechos o expectativas de revalorización o rentabilidad, o derechos parecidos a acciones, obligaciones u otros instrumentos financieros. Incluso entiende que, en caso de utility tokens, si existe una expectativa de revalorización o es susceptible de negociación en mercados semejantes a los de valores (casos en los que el utility token, además de servir para acceder o usar un servicio, o como medio de pago, además «cotiza»), también sería aplicable esta normativa. En todo caso, si la ICO va dirigida a menos de 150 inversores, la inversión mínima es de 100.000 euros y no supera los 5 millones de euros, no se exigiría folleto.

\subsection{Febrero 2018. Comunicado conjunto de la CNMV y del Banco de España sobre criptomonedas y «ofertas iniciales de criptomonedas" (ICO)}

La CNMV y el BdE han comunicado que, «hasta la fecha, ninguna emisión de "criptomoneda" ni ninguna ICO ha sido registrada, autorizada o verificada por ningún organismo supervisor en España. Esto implica que no existen "criptomonedas" ni "tokens" emitidos en ICO cuya adquisición o tenencia en España pueda beneficiarse de ninguna de las garantías o protecciones previstas en la normativa relativa a productos bancarios o de inversión».

En el comunicado, la CNMV y el BdE señalan las dos categorías de «tokens» reconocidas ya a nivel global y en las que se deben encuadrar las distintas ICO que se quieran promover. De esta forma, distinguen entre los security tokens y los utility tokens.

Recogen una serie de consideraciones que deben ser tenidas en cuenta por quien reflexione acerca de participar en una ICO. Las enumeramos: se trata de espacio no regulado, el carácter transfronterizo del fenómeno implica riesgos, existe riesgo de pérdida de la inversión, han de tenerse en cuenta la liquidez y volatilidad y cabe la posibilidad de que la información proporcionada al inversor sea inadecuada.

\subsection{Febrero 2018. EI DOUE publica la Resolución del Parlamento Europeo, de 26 de mayo de 2016, sobre monedas virtuales}

A pesar de no provenir de una autoridad supervisora, entendemos conveniente referir la publicación en el DOUE del pasado 28 de febrero de 2018 (C 76/76 y ss.) de la Resolución 
del Parlamento Europeo, de 26 de mayo de 2016, sobre monedas virtuales (2016/2007(INI)) (2018/C 076/13).

Dedica su última parte a la «regulación inteligente con miras a fomentar la innovación y garantizar la integridad». Y dicha inteligencia se refiere al equilibrio entre el estímulo de las oportunidades y el control de los riesgos.

En lo que interesa al tema que estamos tratando, destacamos el contenido del último párrafo, que refiere la importancia de la formación del consumidor, de la transparencia y de la confianza al utilizar las monedas virtuales. Pide a la Comisión que desarrolle, en cooperación con los Estados miembros y las empresas del sector, directrices destinadas a garantizar que se brinde una información precisa, clara y completa a los usuarios, actuales y futuros, de monedas virtuales, con el fin de permitirles tomar decisiones con pleno conocimiento de causa, lo que mejoraría la transparencia de los sistemas de monedas virtuales en lo que respecta a su organización y funcionamiento y a lo que los diferencia de los sistemas de pago regulados y supervisados en términos de protección del consumidor.

\subsection{Julio 2018. Ampliación del documento de preguntas y respuestas dirigidas a empresas «fintech» sobre actividades y servicios que pueden tener relación con la CNMV}

A través de la pregunta 6.4, la CNMV aclara que bastará con que una entidad autorizada para prestar servicios de inversión valide y supervise de modo general la oferta a inversores y el procedimiento de colocación de criptomonedas.

La CNMV establece que la entidad autorizada debe asegurarse de que la información ofrecida sea "clara, imparcial y no engañosa» y se refiera a las características y riesgos de los valores emitidos, así como a la situación jurídica y económico-financiera del emisor de una manera suficientemente detallada para permitir que el inversor pueda tomar una decisión de inversión fundada.

De este modo el supervisor bursátil disipa las dudas sobre el alcance con el que debe intervenir una entidad autorizada para prestar servicios de inversión en ciertas ofertas dirigidas al público en general empleando cualquier forma de comunicación publicitaria.

Una opción es que la entidad autorizada para prestar servicios de inversión intervenga con ocasión de cada suscripción o adquisición de los valores, o instrumentos financieros de que se trate, como colocadora, intermediaria o asesora. Otra opción es que una entidad autorizada para prestar servicios de inversión valide o supervise de modo general la oferta.

Con carácter general, la CNMV considera que a este tipo de plataformas se les deberían aplicar, como mínimo, normas de custodia o registro, de gestión de conflictos de interés entre clientes y de transparencia en materia de comisiones, además de la normativa de prevención del blanqueo de capitales. Se hace la recomendación de que estas plataformas apliquen de forma voluntaria los principios de la normativa del mercado de valores relativos a las materias señaladas con el fin de garantizar un correcto funcionamiento de su actividad. 


\subsection{Septiembre 2018. La CNMV publica criterios en relación con las ICO}

Con fecha 20 de septiembre, la CNMV hace público el documento que recoge los criterios iniciales de actuación que está aplicando en relación con las ICO. Advierte de que dada la complejidad y novedad del fenómeno, estos criterios están sujetos a revisión en función de la experiencia que se vaya acumulando y el debate que al respecto se está desarrollando actualmente a escala internacional y, en particular, en la AEVM.

Abarca cuatro cuestiones fundamentales: la consideración de los «tokens» como valores negociables, necesidad y alcance de la intervención de entidades autorizadas, representación de «tokens» y sus consecuencias y necesidad de folleto informativo.

En cuanto a la primera cuestión, consideración de los «tokens» como valores negociables, la CNMV establece que se analizarán las propuestas caso por caso. Hace referencia a los criterios establecidos en el comunicado de la CNMV publicado en febrero 2018, pero lo matiza al estimar adecuado excluir de la consideración como valor negociable aquellos casos en los que no quepa razonablemente establecer una correlación entre las expectativas de revalorización o de rentabilidad del instrumento y la evolución del negocio o proyecto subyacente.

En relación con la intervención de entidad autorizada a la que se refiere el artículo 35.3 del TRLMV, se aplicará lo publicado en el documento de preguntas y respuestas de «fintech», también mencionado en párrafos anteriores.

Añade que se considera adecuado que la entidad autorizada no proceda a validar la información que se va a entregar a los inversores, a no ser que en la misma se incluyan advertencias destacadas acerca de la naturaleza novedosa de la tecnología de registro y del hecho de que la custodia de los instrumentos no sea realizada por una entidad habilitada para prestar servicios de inversión.

Considera que no es necesaria, en principio, la intervención de una entidad autorizada para realizar la colocación de los valores ni para la custodia de los valores, con fundamento en la exigencia legal de carácter profesional.

En materia de representación de «tokens» y las consecuencias que lleva aparejadas, recuerda que el artículo 6.1 del TRLMV permite interpretar que es posible que ciertos valores no se representen por medio de anotaciones en cuenta o títulos, por tanto, no puede excluirse la posibilidad del registro de derechos que puedan tener la consideración de valor negociable por medio de la tecnología Blockchain.

Explica que si los «tokens» van a ser negociados en mercados no españoles será la ley (y la autoridad competente) del país donde se encuentre el mercado en el que vayan a negociarse los «tokens» la que determine en qué medida es exigible una forma concreta de representación de los valores para su negociación en un mercado organizado y, en su caso, la necesidad de que el registro lo lleve un depositario central de valores.

Entiende que no parece posible la negociación de los «tokens» en mercados regulados, sistemas multilaterales de negociación o sistemas organizados de contratación españoles ya que el artículo 6.2 del TRLMV exigiría que los «tokens» estén representados por medio de anotaciones en cuenta y el artículo 8.3 del TRLMV obligaría a que el registro lo realizara un depositario central de valores. 
Tampoco parece posible generar un mercado interno en una plataforma no regulada o que los «tokens» se negocien en una plataforma localizada en España ya que estas plataformas deberían contar con las autorizaciones para ejercer su actividad y les serían exigibles la representación mediante anotaciones en cuenta y la participación de un depositario central de valores.

Por último, en lo que se refiere a necesidad de folleto informativo, dado que la mayoría de las operaciones que se están planteando pueden ampararse en el artículo 35.2 del TRLMV (relativo a las situaciones en las que no existe obligación de publicar un folleto), se aconseja a los emisores que se atengan a los criterios comentados anteriormente en relación con el artículo 35.3. Es decir, se aplicará lo publicado en las Q\&A de «fintech» respecto del grado mínimo de intervención de la entidad autorizada para prestar servicios de inversión a efectos de la comercialización (que implica que dicha entidad debe realizar una supervisión general del proceso y validar la información que se va a entregar a los inversores, que deberá ser clara, imparcial y no engañosa y referirse a las características y riesgos de los valores emitidos, así como a la situación jurídica y económico-financiera del emisor de una manera suficientemente detallada para permitir que el inversor pueda tomar una decisión de inversión fundada).

Dadas las dificultades con las que puede encontrarse una ICO para la elaboración de un folleto, cuando resulte preciso por las características de la operación, la CNMV hará el esfuerzo necesario de adaptación y tendrá en cuenta el principio de proporcionalidad (máxime cuando es previsible que las operaciones no sean de gran tamaño) a efectos de reducir en lo posible la complejidad y extensión del documento.

\subsection{Noviembre 2018. Estudio sobre el impacto en la competencia de las nuevas tecnologías en el sector financiero (Fintech) de la Comisión Nacional de los Mercados y la Competencia}

La Comisión Nacional de los Mercados y la Competencia (en adelante, CNMC) hizo público en el mes de noviembre un estudio sobre el impacto en la competencia de las nuevas tecnologías en el sector financiero. En él se pueden encontrar una serie de recomendaciones en la materia, precedidas por un análisis global y otro concreto de este fenómeno disruptivo.

En sede de tecnologías de registro distribuido, refiere y define el fenómeno ICO que nos ocupa, susceptible de ser puesto en relación con carácter pro-competitivo de las entidades «fintech» y las consideraciones relativas a la corrección de fallos de mercado y la revisión de la regulación. En este sentido la autoridad de la competencia española considera que las asimetrías de información pueden mitigarse mediante una mayor transparencia, mientras que la estabilidad financiera se puede alcanzar mediante la prevención de conflictos de intereses.

Además, las recomendaciones efectuadas pueden resumirse así: acomodación al fenómeno, necesidad de llevar a cabo la revisión de la regulación, apostar por la regulación de las actividades y no de entidades, evitar reservas de actividad, aprovechar RegTech para reducir las cargas que implica la regulación, crear un regulatory sandbox y adoptar el principio 
de neutralidad tecnológica, siendo la interoperabilidad y la no discriminación los ejes que podrían marcar el camino regulatorio que se debe seguir.

\section{Conclusiones}

1. Los 40 años de vida de la Constitución española, que apenas tiene un contenido financiero explícito, han coincidido con un periodo de importantes transformaciones en la economía española y, en particular, en su sistema financiero. Como ha sido expuesto, la relación del sistema financiero y su evolución con nuestra Carta Magna, se ha encontrado desde sus inicios intermediada por atribuciones dadas a otras vertientes del ordenamiento jurídico, entre las que destaca las relativas al Derecho comunitario como máxima expresión de apertura económica y jurídica.

2. En este trabajo, en el que se ha tratado de analizar la repercusión jurídica de nuevos fenómenos financieros, concretamente el de las ICO, se ha podido observar que la actitud de los organismos supervisores, en ejercicio de sus funciones administrativas, es considerar la necesidad de nuevos instrumentos de regulación adaptados a los fenómenos disruptivos que sean eficaces en la garantía de la protección del inversor y del sistema financiero en su conjunto. Sabemos que a día de hoy no constituyen, con carácter general, supuestos regulados.

3. En nuestra opinión, el inversor debe ser consciente del riesgo asumido, de la naturaleza del producto en el que se invierte. Pero en ningún caso esta advertencia debe estar encaminada desde la perspectiva del temor financiero o jurídico. El temor financiero podrá ser expuesto por quienes estén especializados en esta rama. El temor jurídico que lleve al inversor a entender que su inversión puede ser constitutiva de un acto ilícito encuentra su respuesta en la autonomía de la voluntad de las partes consagrada en el artículo 1255 de nuestro Código Civil, sobre la que hemos reflexionado, con los límites propios de la misma: ley, moral y orden público y la contribución a la protección del inversor las entidades supervisoras tienen adjudicadas en base a las atribuciones legales.

\section{Bibliografía}

García Rodríguez, A. (2017). «Initial Coin Offering. El nuevo método de financiación nacido de la globalización y la revolución tecnológica». Revista Derecho del Mercado de Valores, núm. 21, versión online.

González Jiménez, P. M. (2018). «La CNMC publica un estudio sobre las nuevas tecnologías en el sector financiero (Fintech)». Blog Derecho de Nuevas Tecnologías, Universidad Pablo de Olavide, recuperado de: https://www.upo.es/investiga/derecho-nuevas-tecnologias/detalle-noticias/La-CNMCpublica-un-estudio-sobre-las-nuevas-tecnologias-en-el-sector-financiero-Fintech/

Ibáñez Jiménez, J. W. (2001). La contratación en el mercado de valores, Madrid: Marcial Pons.

Muñoz Pérez, A. F. (2018). «Crowdfunding en Europa; entre la fragmentación y la unidad de mercado». Revista Derecho del Mercado de Valores, núm. 22, versión online.

Ontiveros Baeza, E. y Valero López, F. J. (2003). «El sistema financiero español desde la Constitución. Homologación internacional, vertebración territorial». Economía industrial, núm. 349, págs. 111126.

Pastor Sempere, C. (2017). «Criptodivisas; ¿una disrupción jurídica en la eurozona?». Revista de Estudios Europeos, núm. 70, págs. 284-318. 
- (2017). «La estandarización de la información financiera de pymes y autónomos como clave de acceso a la financiación». Revista de derecho bancario y bursátil, núm. 146, págs. 179-205.

Ramos Suárez, F. M. (2018). «La digitalización del sector financiero "tokens" e ICO (Initial Coin Oferings)». Revista Derecho del Mercado de Valores, núm. 22, versión online.

\section{Otras fuentes consultadas (por orden de aparición):}

Mi Ethereum (2018). «¿Qué es una ICO?». Recuperado de: https://miethereum.com/blockchain/ico/.

Comisión Nacional del Mercado de Valores (2017). «Comunicado ESMA alerta a los inversores por el alto riesgo de las ICO». Recuperado de: http://www.cnmv.es/Portal/verDoc.axd?t=\%7be74d1624dbc2-46fb-93ef-e4c8d4e2cdc0\%7d.

Autoridad Europea de Valores y Mercados (2017). «ICOs». Recuperado de: https://www.esma.europa. eu/search/site/icoS.

Comisión Nacional del Mercado de Valores (2017). "Comunicado ESMA alerta a las entidades que participen en ICOs sobre la necesidad de conocer requisitos regulatorios a los que deben estar sometidos». Recuperado de: http://www.cnmv.es/Portal/verDoc.axd?t=\%7bb523a876-9f2a-42caaca7-791e5e16fc30\%7d.

Comisión Nacional del Mercado de Valores (2018). "Comunicado difundido por la Securities and Exchange Commission con consideraciones de su presidente sobre las criptomonedas y las denominadas "Initial Coin Offerings" o "ICOs"». Recuperado de: https://www.cnmv.es/portal/ verDoc.axd? $t=\{14 a 617 e 8-7 f 18-40 e 0-9 f 1 b-2061 d 924 f 5 f 4\}$.

Comisión Nacional del Mercado de Valores (2018). "Consideraciones de la CNMV sobre "criptomonedas" e "ICOs" dirigidas a los profesionales del sector financiero". Recuperado de: http://www.cnmv.es/ Portal/verDoc.axd?t=\%7b9c76eef8-839a-4c19-937f-cfde6443e4bc\%7d.

Comisión Nacional del Mercado de Valores y Banco de España (2018). "Comunicado conjunto de la CNMV y del Banco de España sobre "criptomonedas" y "ofertas iniciales de criptomonedas" (ICOs)». Recuperado de: https://www.bde.es/f/webbde/GAP/Secciones/SalaPrensa/NotasInformativas/18/ presbe2018_07.pdf.

Comisión Nacional del Mercado de Valores (2018). «Preguntas y respuestas dirigidas a empresas FinTech sobre actividades y servicios que puedan tener relación con la CNMV». Recuperado de: http://cnmv.es/docportal/legislacion/faq/qasfintech.pdf.

Comisión Nacional del Mercado de Valores (2018). «Criterios en relación con las ICOs». Recuperado de: http://cnmv.es/DocPortal/Fintech/CriteriosICOs.pdf.

Comisión Nacional de los Mercados y la Competencia (2018). «Estudio sobre el impacto en la competencia de las nuevas tecnologías en el sector financiero (Fintech)". Recuperado de: https://www.cnmc. es/node/371812. 